\title{
Thoracoscopic management of chylothorax after esophagectomy
}

\author{
C. Callari $\cdot$ S. Perretta $\cdot$ M. Diana $\cdot$ \\ J. Dagostino $\cdot$ B. Dallemagne $\cdot$ J. Marescaux
}

Received: 30 March 2011/ Accepted: 30 September 2011/Published online: 15 November 2011

(C) Springer Science+Business Media, LLC 2011

\begin{abstract}
Background Chylothorax after esophagectomy is a potentially life-threatening complication, with a reported incidence rate of $1-4 \%$. Two cases of postoperative chylothorax successfully managed thoracoscopically are reported. Methods In case 1, a 61-year-old man presenting with an adenocarcinoma of the lower esophagus underwent laparoscopic transhiatal esophagectomy after neoadjuvant chemotherapy. The thoracic duct was identified, and no obvious leaks were detected. The thoracic drain was removed on postoperative day (POD) 6, and chest X-rays were normal. The patient was discharged on POD 10. On POD 20, he was readmitted for acute cardiopulmonary distress. Computed tomography scan showed a massive right collection. After insertion of a chest tube, 81 of chylous fluid were drained. Once hemodynamic stabilization had been established, the patient was scheduled for surgery. In case 2, a 54-year-old woman presenting with esophageal stenosis after caustic injury refractory to balloon dilation and stenting underwent esophagectomy. Mediastinal dissection was difficult due to fibrotic reaction. On POD 2, the patient presented with a massive chylothorax. In both cases, three trocars were inserted in the right pleural cavity. An incomplete lateral injury of the
\end{abstract}

thoracic duct was found in case 1, and a complete transection proximal to the cervical anastomosis next to the left subclavian was found in case 2. Clips and sutures were used first to seal the duct. Fibrin glue was applied to reinforce the closure. A chest tube was left in place.

Results The operative time was $60 \mathrm{~min}$ in case 1 and 55 $\min$ in case 2 . The chylothorax did not recur, although the postoperative course was longer in case 2 due to associate comorbidities.

Conclusions The thoracic duct is exposed to injuries during esophagectomies, especially in cases of cancer and postcaustic injuries, leading to fibrotic reaction of the surrounding tissue. Early and delayed chylothorax can be managed efficiently by a thoracoscopic approach replicating the sealing techniques used in thoracotomy.

Keywords Chylothorax · Esophagectomy ·

Thoracoscopy

Disclosures C. Callari, S. Perretta, M. Diana, J. Dagostino, B. Dallemagne, and J. Marescaux have no conflicts of interest of financial ties to disclose.
Electronic supplementary material The online version of this article (doi:10.1007/s00464-011-1988-3) contains supplementary material, which is available to authorized users.

C. Callari $(\bowtie) \cdot$ S. Perretta $\cdot$ M. Diana · J. Dagostino ·

B. Dallemagne $\cdot$ J. Marescaux

IRCAD, Department of Endocrine and Digestive Surgery,

University of Strasbourg, Strasbourg, France

e-mail: cosimo.callari@chru-strasbourg.fr 